Pacific Journal of Mathematics

LINEAR EIGENVALUES AND A NONLINEAR BOUNDARy

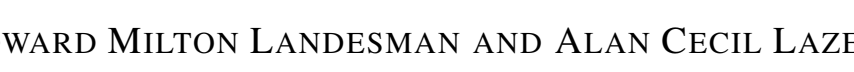




\title{
LINEAR EIGENVALUES AND A NONLINEAR BOUNDARY VALUE PROBLEM
}

\author{
E. M. Landesman AND A. C. Lazer
}

In this paper a nonlinear boundary value problem for elliptic partial differential equations is considered. The principal result generalizes a previous result on a two point boundary value problem for a nonlinear second order ordinary differential equation. The solvability condition obtained for the nonlinear problem is related to the eigenvalues of an associated linear problem.

In [5] the second author and D. E. Leach considered the two point boundary value problem

$$
\begin{aligned}
& u^{\prime \prime}+p\left(x, u, u^{\prime}\right) u=h\left(x, u, u^{\prime}\right) \\
& u(0)=a, u(\pi)=b .
\end{aligned}
$$

It was shown that, if for some integer $N$ there exist numbers $\gamma_{N}$ and $\gamma_{N+1}$ such that

$$
N^{2}<\gamma_{N} \leqq p(x, s, r) \leqq \gamma_{N+1}<(N+1)^{2},
$$

if $p(x, s, r)$ and $h(x, s, r)$ are continuous on $[0, \pi] \times(-\infty, \infty) \times(-\infty, \infty)$, and $h$ is bounded, then the problem (1.1) has at least one solution.

In this paper we consider the $n$-dimensional analogue of the problem (1.1) which is

$$
\begin{gathered}
\Delta u+p\left(x, u, \frac{\partial u}{\partial x_{1}}, \cdots, \frac{\partial u}{\partial x_{n}}\right) u=h\left(x, u, \frac{\partial u}{\partial x_{1}}, \cdots, \frac{\partial u}{\partial x_{n}}\right), \\
u(x)=g(x) \text { on } \partial D,
\end{gathered}
$$

where $D$ is a domain in $R^{n}$ and $\Delta$ is the $n$-dimensional Laplacian. The corresponding result is that if $D$ is a Dirichlet domain, if there exist numbers $\gamma_{N}$ and $\gamma_{N+1}$ such that

$$
\alpha_{N}<\gamma_{N} \leqq p\left(x, t, s_{1}, \cdots, s_{n}\right) \leqq \gamma_{N+1}<\alpha_{N+1},
$$

for $\left(x, t, s_{1}, \cdots, s_{n}\right) \in \bar{D} \times R^{n+1}$ where

$$
\alpha_{1} \leqq \alpha_{2} \leqq \cdots \leqq \alpha_{K} \leqq \alpha_{K+1} \leqq \cdots,
$$

are the eigenvalues of the problem

$$
\Delta u+\alpha u=0, \quad u=0 \text { on } \partial D,
$$

if $p\left(x, t, s_{1}, \cdots, s_{n}\right), h\left(x, t, s_{1}, \cdots, s_{n}\right)$ are continuous and $h$ bounded on 
$\bar{D} \times R^{n}$, then for any continuous $g(x)$ there exists a function $v$ continuously differentiable on $\bar{D}$ with $v(x)=g(x)$ on $\partial D$ which satisfies (1.2) in the distribution sense.

With the exception of the Schauder fixed point theorem the methods used in [5] were very elementary. The methods in this paper are similar to those of [5] but rely strongly on the spectral theory of symmetric completely continuous operators and variational properties of eigenvalues.

In the second section we consider the linear homogeneous problem

$$
L u+p(x) u=h(x), \quad u=0 \text { on } \partial D
$$

where $L$ is a strongly elliptic self adjoint operator and $D \subset R^{n}$ is an arbitrary domain. Under suitable conditions on $p$ and $h$ we obtain an a priori bound for solutions of (1.4).

Using this result and Schauder's method we obtain an existence theorem for the problem

$$
\begin{gathered}
L u+p\left(x, u, \frac{\partial u}{\partial x_{1}}, \cdots, \frac{\partial u}{\partial x_{n}}\right) u=h\left(x, u, \frac{\partial u}{\partial x_{1}}, \cdots, \frac{\partial u}{\partial x_{n}}\right), \\
u=0 \text { on } \partial D .
\end{gathered}
$$

The aforementioned result follows quickly from this theorem.

A special case of our principal result follows in a straight-forward way from a result on Hammerstein integral equations due to C. L. Dolph [4]. Namely, if $F(x, t)$ is continuously differentiable in $t$ and $x$ and continuous for $(x, t) \in \bar{D} \times R$, if $h(x)$ is continuously differentiable on $\bar{D}$, and

$$
\alpha_{N}<\gamma_{N} \leqq \frac{\partial F}{\partial t}(x, t) \leqq \gamma_{N+1}<\alpha_{N+1}
$$

then the problem

$$
\Delta u+F(x, u)=h(x), \quad u=g \text { on } \partial D,
$$

$g$ continuous, has a unique solution in the classical sense.

To see that this problem is included in the problem (1.2) we note that the differential equation can be written

$$
\begin{gathered}
\Delta u+p(x, u) u=\hat{h}(x), \text { where } \\
p(x, u)=\int_{0}^{1} \frac{\partial F}{\partial t}(x, s u) d s, \hat{h}(x)=h(x)-F(x, 0) .
\end{gathered}
$$

Clearly $\gamma_{N} \leqq p(x, t) \leqq \gamma_{N+1}$.

2. Preliminaries. In this section we recall briefly results con- 
cerning elliptic differential equations, completely continuous operators, and variational properties of eigenvalues in forms applicable to our problem. We also give an auxiliary lemma which is applied in the next section.

Let $D$ be a bounded domain in $R^{n}$. In the following $L$ will denote a second-order, self adjoint, strongly elliptic differential operator involving only principal part. That is, a formal expression

$$
L=\sum_{i=1}^{n} \sum_{j=1}^{n} \frac{\partial}{\partial x_{i}} a^{i j} \frac{\partial}{\partial x_{j}}
$$

where for $i, j=1, \cdots, n, a^{i j}=a^{j i}$ is a real valued function bounded and measurable on $D$ and there exists a constant $c>0$ such that for all $x \in D$

$$
\sum_{i=1}^{n} \sum_{j=1}^{n} a^{i j}(x) \xi_{i} \xi_{j} \geqq c \sum_{i=1}^{n} \xi_{i}^{2}
$$

for arbitrary real numbers $\xi_{1}, \cdots, \xi_{n}$.

Let $H_{0}$ denote real $L^{2}(D)$ and if $f, g \in H_{0}$ let

$$
\langle f, g\rangle_{0}=\int_{D} f g d x \text {. }
$$

More generally if $p$ is a real measurable function defined on $D$ such that there are numbers $\delta>0$ and $\Delta$ with

$$
0<\delta \leqq p(x) \leqq \Delta
$$

for all $x \in D$, let

$$
\langle f, g\rangle_{0, p}=\int_{D} p f g d x .
$$

Clearly \langle\rangle$_{0, p}$ defines an inner product on $H_{0}$ which induces the same topology on $H_{0}$ as $\langle,\rangle_{0}$.

Let $\dot{C}_{1}$ denote the inner product space of real continuously differentiable functions defined on $R^{n}$ and having compact support contained in $D$ with real inner product given by

$$
\langle u, v\rangle_{1}=\int_{D}\left[u v+\sum_{i=1}^{n}\left(\frac{\partial u}{\partial x_{i}}\right)\left(\frac{\partial v}{\partial x_{i}}\right)\right] d x
$$

for $u, v \in \dot{C}_{1}$. Let $B$ be the symmetric bilinear form defined on $\dot{C}_{1}$ by

$$
B(u, v)=\int_{D} \sum_{i=1}^{n} \sum_{j=1}^{n} a^{i j}\left(\frac{\partial u}{\partial x_{i}}\right)\left(\frac{\partial v}{\partial x_{j}}\right) d x .
$$

The boundedness of the $a^{i j}$, the strong ellipticity condition (2.1), and Poincare's inequality [1, p. 73] imply the existence of positive constants $K_{1}, K_{2}$, and $K_{3}$ such that for all $u \in \dot{C}_{1}$ 


$$
\begin{gathered}
K_{1}\langle u, u\rangle_{0} \leqq B(u, u), \\
K_{2}\langle u, u\rangle_{1} \leqq B(u, u) \leqq K_{3}\langle u, u\rangle_{1} .
\end{gathered}
$$

Let $\stackrel{\circ}{H}_{1}$ denote the real Hilbert space obtained by completing $\dot{C}_{1}$ with respect to $\langle,\rangle_{1}$. It is known that the underlying space of $\stackrel{\circ}{H}_{1}$ may be assumed to be a subset of $H_{0}([1, \mathrm{p} .2])$. The quadratic form $B$ may be extended by continuity to $\stackrel{\circ}{H}_{1}$ and as a consequence of (2.4) defines an inner product on $\stackrel{H}{1}_{1}$ which induces the same topology on $\stackrel{\circ}{H}_{1}$ as $\langle,\rangle_{1}$.

The following definition connects the operator $L$ and the quadratic form $B$ : Let $f \in H_{0}$. A weak solution of the boundary value problem

$$
L u=-f, \quad u=0 \text { on } \partial D
$$

is a member $v$ of $\stackrel{\circ}{H}_{1}$ such that

$$
B(\varphi, v)=\langle\varphi, f\rangle_{0}
$$

for all $\varphi \in H_{1}^{\circ}$. This definition is motivated by multiplying (2.5) by a member $\varphi$ of $\dot{C}_{1}$ and formally integrating by parts. The additional condition $v=0$ on $\partial D$ "in a weak sense," is interpreted simply to mean $v \in \stackrel{\circ}{H}_{1}$. Since the linear functional $L_{f}$ defined on $\stackrel{\circ}{H}_{1}$ by $L_{f}(\varphi)=$ $\langle\varphi, f\rangle_{0}$ is continuous, by (2.3), it follows by the Riesz-Frechét theorem that there exists a unique $v \in \stackrel{\circ}{H}_{1}$ such that $B(\varphi, v)=L_{f}(\varphi)=\langle\varphi, f\rangle_{0}$ for all $\varphi \in \stackrel{\circ}{H}_{1}$. Hence there exists a unique weak solution of (2.5).

More generally if $p$ is a function which satisfies a condition of the form (2.2) and $f \in \stackrel{\circ}{H}_{1}$ then the linear functional $L_{p, f}$ defined on $\stackrel{\circ}{H}_{1}$ by $L_{p, f}(\varphi)=\langle\varphi, f\rangle_{0, p}$ is continuous so there exists a unique $T_{p} f \in \stackrel{\circ}{H}_{1}$ such that

$$
B\left(\varphi, T_{p} f\right)=L_{p, f}(\varphi)=\langle\varphi, f\rangle_{0, p}
$$

for all $\varphi \in \stackrel{\circ}{H}_{1}$. This defines a linear map $T_{p}: H_{0} \rightarrow \stackrel{\circ}{H}_{1}$ but since $\stackrel{\circ}{H}_{1} \subset H_{0}$ we may consider $T_{p}$ as a linear map from $H_{0}$ into $H_{0}$. As a consequence of (2.2) and (2.3) it follows that $T_{p}$ is continuous and maps bounded subsets of $H_{0}$ into bounded subsets of $H_{1}^{\circ}$. Thus, by Rellich's selection principle $\left[1\right.$, p. 30], $T_{p}: H_{0} \rightarrow H_{0}$ is completely continuous. Moreover $T_{p}$ is symmetric and positive with respect to the $\langle,\rangle_{0, p}$ inner product. Indeed, if $f, g \in H_{0}$, then by taking $\varphi$ to be $T_{p} f$ and $T_{p} g$ in (2.7) we obtain

$$
\begin{aligned}
\left\langle T_{p} f, g\right\rangle_{0, p} & =B\left(T_{p} f, T_{p} g\right)=B\left(T_{p} g, T_{p} f\right) \\
& =\left\langle T_{p} g, f\right\rangle_{0, p}=\left\langle f, T_{p} g\right\rangle_{0, p} .
\end{aligned}
$$

If for some $f \in H_{0}, T_{p} f=0$ then $\langle\varphi, f\rangle_{0, p}=0$ for all $\varphi \in \dot{C}_{1}$ and since 
$\dot{C}_{1}$ is dense in $H_{0}, f=0$. Thus if $f \in H_{0}$ it follows from (2.7) by taking $\varphi=T_{p} f \in \stackrel{\circ}{H}_{1}$ that $\left\langle T_{p} f, f\right\rangle_{0, p}=B\left(T_{p} f, T_{p} f\right)$ so

$$
\left\langle T_{p} f, f\right\rangle_{0, p}>0 \text { if } f \neq 0 \text {. }
$$

Applying the results of $\S 93$ and $\S 94$ of [7] to $T_{p}$, we infer the existence of a sequence of real numbers $\left\{\lambda_{k}\right\}_{1}^{\infty}$ and a sequence $\left\{\varphi_{k k}\right\}_{1}^{\infty}$ in $H_{0}$ such that:

$$
\begin{gathered}
\varphi_{k}=\lambda_{k} T_{p} \varphi_{k} \\
\left\langle\varphi_{k}, \varphi_{j}\right\rangle_{0, p}=\delta_{k j}=\left\{\begin{array}{l}
0 ; k \neq j \\
1 ; k=j
\end{array}\right. \\
T_{p} f=\sum_{k=1}^{\infty} \frac{\left\langle f, \varphi_{k}\right\rangle_{0, p}}{\lambda_{k}} \varphi_{k}
\end{gathered}
$$

for all $f \in H_{0}$, and if $\mu \neq \lambda_{k}$ for all $k=1,2 \cdots$ the mapping $\left[I-\mu T_{p}\right]$ : $H_{0} \rightarrow H_{0}$ is bijective and has a continuous inverse defined by

$$
\left[I-\mu T_{p}\right]^{-1} g=g+\mu \sum_{k=1}^{\infty} \frac{\left\langle g, \varphi_{k}\right\rangle_{0, p}}{\lambda_{k}-\mu} \Phi_{k} .
$$

Moreover, the sequence $\left\{\lambda_{k}\right\}$ has no finite cluster point so we may assume by (2.8) that

$$
0<\lambda_{1} \leqq \lambda_{2} \leqq \cdots \leqq \lambda_{k} \leqq \lambda_{k+1} \leqq \cdots .
$$

Using (2.7) and (2.9) we obtain

$$
B\left(\theta, \varphi_{k}\right)=\left\langle\theta, \lambda_{k} \varphi_{k}\right\rangle_{0, p}=\left\langle\theta, \lambda_{k} p \varphi_{k}\right\rangle_{0}
$$

for all $\theta \in \dot{H}_{1} \quad$ Hence, for each $k=1,2, \cdots, \varphi_{k}$ is a nontrivial weak solution of the boundary value problem

$$
L u+\lambda_{k} p(x) u=0, \quad u=0 \text { on } \partial D .
$$

We therefore call $\lambda_{k}$ a weak eigenvalue corresponding to $p$.

In the following we will want to consider different functions which satisfy a condition of the form (2.2) so henceforth we write $\lambda_{k}=\lambda_{k}(p), k=1,2, \cdots$.

A result in $\S 93$ of [7] and (2.8) implies that the sequence $\left\{\varphi_{k}\right\}_{1}^{\infty}$ is complete in $H_{0}$ so Parseval's formula

$$
(f, f)_{0, p}=\sum_{k=1}^{\infty}\left\langle f, \varphi_{k}\right\rangle_{0, p}^{2}
$$

holds for all $f \in H_{0}$. We now derive a similar identity involving the space $\stackrel{\circ}{H}_{1}$ and the inner product $B$.

Using (2.7), (2.9), and (2.10) we have 


$$
B\left(\varphi_{k}, \varphi_{j}\right)=B\left(\lambda_{k}(p) T_{p} \varphi_{k}, \varphi_{j}\right)=\lambda_{k}(p)\left\langle\varphi_{k}, \varphi_{j}\right\rangle_{0, p}=\lambda_{k}(p) \delta_{k j}
$$

which shows that the sequence $\left\{\left(1 / \sqrt{\lambda_{k}(p)}\right) \varphi_{k}\right\}_{1}^{\infty}$ in $\stackrel{\circ}{H}_{1}$ is orthonormal with respect to the inner product $B$. If for some $\theta \in \stackrel{\circ}{H}_{1}, B\left(\varphi_{k}, \theta\right)=0$ for all $k$ then by (2.7) and (2.9),

$$
\left\langle\varphi_{k}, \theta\right\rangle_{0, p}=0 \text { for all } k,
$$

so $\theta=0$. Thus the sequence $\left\{\left(1 / \sqrt{\lambda_{k}(p)}\right) \varphi_{k}\right\}$ is complete in $\dot{H}_{1}$ and by Parseval's formula

$$
B(\theta, \theta)=\sum_{k=1}^{\infty} B\left(\frac{1}{\sqrt{\lambda_{k}(p)}} \varphi_{k}, \theta\right)^{2} .
$$

Using (2.7) and (2.9) we may rewrite this in the more convenient form

$$
B(\theta, \theta)=\sum_{k=1}^{\infty} \lambda_{k}(p)\left\langle\theta, \varphi_{k}\right\rangle_{0, p}^{2} .
$$

The identities (2.14) and (2.15) together with (2.13) now yield the following variational characterization of the weak eigenvalues in terms of the inner products $B$ and \langle\rangle$_{0, p}$ :

$$
\begin{aligned}
\lambda_{1}(p) & =\min \left\{B(\theta, \theta) \mid \theta \in \stackrel{\circ}{H}_{1},\langle\theta, \theta\rangle_{0, p}=1\right\} \\
\lambda_{k+1}(p) & =\min \left\{\begin{array}{l}
B(\theta, \theta) \mid \theta \in \stackrel{\circ}{H}_{1},\langle\theta, \theta\rangle_{0, p}=1, \\
\langle\theta, \varphi\rangle_{0, p}=0 ; j=1, \cdots, k
\end{array}\right\} .
\end{aligned}
$$

Indeed if $\theta \in \stackrel{\circ}{H}_{1},\langle\theta, \theta\rangle_{0, p}=1$ and $\left\langle\theta, \varphi_{j}\right\rangle_{0, p}=0$ for all $j=1, \cdots, k$ then by (2.13), (2.14) and (2.15)

$$
\begin{aligned}
B(\theta, \theta) & =\sum_{m=k+1}^{\infty} \lambda_{m}(p)\left\langle\theta, \varphi_{m}\right\rangle_{0, p}^{2} \geqq \lambda_{k+1}(p) \sum_{m=k+1}^{\infty}\left\langle\theta, \varphi_{m}\right\rangle_{0, p}^{2} \\
& =\lambda_{k+1}(p)\langle\theta, \theta\rangle_{0,}=\lambda_{k+1}(p),
\end{aligned}
$$

while $B\left(\varphi_{k+1}, \varphi_{k+1}\right)=\lambda_{k+1}$. The verification of the first identity in (2.16) is similar.

The proofs of the following two lemmas are essentially the same as the proofs given for similar results in [2, Chapter 6].

Lemma 2.1 (Courant). If for $v_{1}, \cdots, v_{k}$ in $H_{0}$ one defines

$$
\mu_{k}(p)\left(v_{1}, \cdots, v_{k}\right)=\inf \left\{\begin{array}{l}
B(\theta, \theta) \mid \theta \in \stackrel{\circ}{H}_{1},\langle\theta, \theta\rangle_{0, p}=1, \\
\left\langle\theta, v_{j}\right\rangle_{0, p}=0 ; j=1, \cdots, k
\end{array}\right\}
$$

then

$$
\lambda_{k+1}(p)=\sup \left\{\begin{array}{l}
\mu_{k}(p)\left(v_{1}, \cdots, v_{k}\right) \mid v_{j} \in H_{0}, \\
j=1, \cdots, k
\end{array}\right\}
$$


Proof. Since we have established above that $\lambda_{k+1}(p)=\mu_{k}(p)\left(\varphi_{1}\right.$, $\cdots, \varphi_{k}$ ) we need only show that for arbitrary $v_{1}, \cdots, v_{k} \in H_{0}$

$$
\mu_{k}(p)\left(v_{1}, \cdots, v_{k}\right) \leqq \lambda_{k+1}(p) .
$$

Given $v_{1}, \cdots, v_{k} \in H_{0}$ let $c_{j}, j=1, \cdots, k+1$ be numbers such that $\sum_{j=1}^{k+1} c_{j}\left\langle\varphi, v_{i}\right\rangle_{0, p}=0$ for $i=1, \cdots, k$ and $\sum_{j=1}^{k+1} c_{j}^{2}=1$. If $\theta=\sum_{j=1}^{k+1} c_{j} \varphi_{j}$ then $\left\langle\theta, v_{i}\right\rangle_{0, p}=0 ; i=1, \cdots, k$ and $\langle\theta, \theta\rangle_{0, p}=1$. Thus from (2.15), $\mu_{k}(p)\left(v_{1}, \cdots, v_{k}\right) \leqq B(\theta, \theta)=\sum_{j=1}^{k+1} c_{j}^{2} \lambda_{j}(p) \leqq \lambda_{k+1}(p) \sum_{j=1}^{k+1} c_{j}^{2}=\lambda_{k+1}(p)$.

This proves (2.17) and hence the lemma.

LEMMA 2.2. If $p$ and $q$ are two real measurable functions defined on $D$ each of which satisfies a condition of the form (2.2) and if for all $x \in D$

$$
p(x) \leqq q(x)
$$

then

$$
\lambda_{j}(q) \leqq \lambda_{j}(p) ; j=1,2, \cdots
$$

Proof. If $v \in H_{0}$ let

$$
\hat{v}(x)=(q(x) / p(x)) v(x) .
$$

Let $v_{1}, \cdots, v_{k} \in H_{0}$ be arbitrary, $k \geqq 1$. We assert that

$$
\mu_{k}(q)\left(v_{1}, \cdots, v_{k}\right) \leqq \mu_{k}(p)\left(\hat{v}_{1}, \cdots, \widehat{v}_{k}\right) .
$$

To prove this inequality we note that if $\varepsilon>0$ it follows from the definition of $\mu_{k}(p)\left(\hat{v}_{1}, \cdots, \widehat{v}_{k}\right)$ that there exists $\theta \in \stackrel{\circ}{H}_{1}$ such that $\langle\theta, \theta\rangle_{0, p}=1,\left\langle\theta, \hat{v}_{j}\right\rangle_{0, p}=0 ; j=1, \cdots, k$, and

$$
B(\theta, \theta) \leqq \mu_{k}(p)\left(\hat{v}_{1}, \cdots, \hat{v}_{k}\right)+\varepsilon .
$$

If $\theta^{*}=\left(1 / \sqrt{\left.\langle\theta, \theta\rangle_{0, q}\right)} \theta\right.$ then using (2.20) we obtain

$$
\left\langle\theta^{*}, v_{j}\right\rangle_{0, q}=\left\langle\theta^{*}, \hat{v}_{j}\right\rangle_{0, p}=0 ; j=1, \cdots, k .
$$

Consequently, since $\left\langle\theta^{*}, \theta^{*}\right\rangle_{0, q}=1$, we have the inequality

$$
\mu_{k}(q)\left(v_{1}, \cdots, v_{k}\right) \leqq B\left(\theta^{*}, \theta^{*}\right) .
$$

Since $B\left(\theta^{*}, \theta^{*}\right)=\left(1 /\langle\theta, \theta\rangle_{0, q}\right) B(\theta, \theta)$, and

$$
\begin{gathered}
1 \leqq\langle\theta, \theta\rangle_{0, p}=\int_{D} p \theta^{2} d x \leqq \int_{D} q \theta^{2} d x=\langle\theta, \theta\rangle_{0, q}, \\
B\left(\theta^{*}, \theta^{*}\right) \leqq B(\theta, \theta) .
\end{gathered}
$$

Combining this inequality with (2.22) and (2.23) we obtain 


$$
\mu_{k}(q)\left(v_{1}, \cdots, v_{k}\right) \leqq \mu_{k}(p)\left(\hat{v}_{1}, \cdots, \widehat{v}_{k}\right)+\varepsilon,
$$

and since $\varepsilon>0$ is arbitrary (2.21) follows.

Now as $v$ ranges over all elements of $H_{0}, \hat{v}$ ranges over all elements of $H_{0}$ and conversely. Hence for $k \geqq 1$, Lemma 2.1 implies

$$
\begin{aligned}
\lambda_{k+1}(q) & =\sup \left\{\begin{array}{l}
\mu_{k}(q)\left(v_{1}, \cdots, v_{k}\right) \mid v_{j} \in H_{0}, \\
j=1, \cdots, k
\end{array}\right\} \\
& \leqq \sup \left\{\begin{array}{l}
\mu_{k}(p)\left(\hat{v}_{1}, \cdots, \hat{v}_{k}\right) \mid v_{j} \in H_{0}, \\
j=1, \cdots, k
\end{array}\right\} \\
& =\sup \left\{\begin{array}{l}
\mu_{k}(p)\left(v_{1}, \cdots, v_{k}\right) \mid v_{j} \in H_{0}, \\
j=1, \cdots, k
\end{array}\right\}=\lambda_{k+1}(p)
\end{aligned}
$$

and this proves (2.19) for $j \geqq 2$. The proof for $j=1$ follows from the first identity of (2.16) and an argument similar to that given above.

In the following lemma the sequence $\left\{\alpha_{k}\right\}_{1}^{\infty}$ will be defined by

$$
\alpha_{k}=\lambda_{k}(1) \quad k=1,2, \cdots
$$

so that each $\alpha_{k}$ is a weak eigenvalue for the problem

$$
L u+\lambda u=0, \quad u=0 \text { on } \partial D .
$$

We let $\gamma_{N}$ and $\gamma_{N+1}$ denote fixed numbers such that for a fixed integer $N$,

$$
\alpha_{N}<\gamma_{N}<\gamma_{N+1}<\alpha_{N+1} \text {. }
$$

$\mathscr{P}\left(\gamma_{N}, \gamma_{N+1}\right)$ will denote the set of functions $p$, measurable on $D$, such that

$$
\gamma_{N} \leqq p(x) \leqq \gamma_{N+1} \text { for all } x \in D
$$

Lemma 2.3. If $h \in H_{0}$ and $p \in \mathscr{P}\left(\gamma_{N}, \gamma_{N+1}\right)$ there exists a unique weak solution of the boundary value problem

$$
L u+p u=h, \quad u=0 \text { on } \partial D .
$$

Moreover there exists a number $M$, independent of $p \in \mathscr{P}\left(\gamma_{N}, \gamma_{N+1}\right)$ such that if $v$ denotes this weak solution then

$$
B(v, v) \leqq M\langle h, h\rangle_{0} \cdot
$$

Proof. The condition that $v$ be a weak solution of (2.27) is equivalent to the condition that for all $\varphi \in \stackrel{\circ}{H}_{1}$

$$
B(\varphi, v)=\langle\varphi, p v-h\rangle_{0}=\langle\varphi, v-h / p\rangle_{0, p}
$$

or by $(2.7)$ that 
$(2.29)$

$$
\left[I-T_{p}\right] v=T_{p}[-h / p] .
$$

By (2.12) this equation can be solved uniquely for $v$ provided that

$$
\lambda_{k}(p) \neq 1 \text { for all } k=1,2, \cdots .
$$

From the inequality (2.26) and Lemma 2.2 we have

$$
\lambda_{k}\left(\gamma_{N+1}\right) \leqq \lambda_{k}(p) \leqq \lambda_{k}\left(\gamma_{N}\right), k=1,2, \cdots .
$$

Clearly, for all $k=1,2, \cdots$,

$$
\begin{gathered}
\lambda_{k}\left(\gamma_{N}\right)=\frac{1}{\gamma_{N}} \lambda_{k}(1)=\frac{\alpha_{k}}{\gamma_{N}}, \\
\lambda_{k}\left(\gamma_{N+1}\right)=\frac{1}{\gamma_{N+1}} \lambda_{k}(1)=\frac{\alpha_{k}}{\gamma_{N+1}},
\end{gathered}
$$

and hence from $(2.25)$,

$$
\begin{aligned}
\lambda_{1}(p) & \leqq \lambda_{2}(p) \leqq \cdots \leqq \lambda_{N}(p) \leqq \lambda_{N}\left(\gamma_{N}\right)=\frac{\alpha_{N}}{\gamma_{N}} \\
& <1<\frac{\alpha_{N+1}}{\gamma_{N+1}}=\lambda_{N+1}\left(\gamma_{N+1}\right) \leqq \lambda_{N+1}(p) \leqq \lambda_{N+2}(p) \leqq \cdots
\end{aligned}
$$

Thus, if

$$
\delta=\min \left[1-\frac{\alpha_{N}}{\gamma_{N}}, \frac{\alpha_{N+1}}{\gamma_{N+1}}-1\right],
$$

then for all $p \in \mathscr{P}\left(\mu_{N}, \mu_{N+1}\right)$,

$$
\left|\lambda_{k}(p)-1\right| \geqq \delta \text { for all } k=1,2, \cdots .
$$

Consequently, (2.29) has a unique solution which by (2.11) and (2.12) is given by

$$
\begin{aligned}
v & =\left[I-T_{p}\right]^{-1} T_{p}\left[-\frac{h}{p}\right]=T_{p}\left[I-T_{p}\right]^{-1}\left[-\frac{h}{p}\right] \\
& =T_{p}\left[-\frac{h}{p}+\sum_{k=1}^{\infty} \frac{\left\langle-h / p, \varphi_{k}\right\rangle_{0, p} \varphi_{k}}{\lambda_{k}(p)-1}\right] \\
& =\sum_{k=1}^{\infty} \frac{\left\langle-h / p, \varphi_{k}\right\rangle_{0, p} \varphi_{k}}{\lambda_{k}(p)}+\sum_{k=1}^{\infty} \frac{\left\langle-h / p, \varphi_{k}\right\rangle_{0, p} \varphi_{k}}{\lambda_{k}(p)\left(\lambda_{k}(p)-1\right)} \\
& =\sum_{k=1}^{\infty} \frac{\left\langle-h / p, \varphi_{k}\right\rangle_{0, p} \varphi_{k}}{\lambda_{k}(p)-1} .
\end{aligned}
$$

Hence, from (2.15) and (2.33) we have 


$$
\begin{aligned}
B(v, v) & =\sum_{k=1}^{\infty} \lambda_{k}(p)\left\langle v, \varphi_{k}\right\rangle_{0, p}^{2}=\sum_{k=1}^{\infty} \frac{\lambda_{k}(p)\left\langle-h / p, \varphi_{k}\right\rangle_{\nu, p}^{2}}{\left[\lambda_{k}(p)-1\right]^{2}} \\
& \leqq \frac{1}{\delta} \sup _{k}\left|\frac{\lambda_{k}(p)}{\lambda_{k}(p)-1}\right| \sum_{k=1}^{\infty}\left\langle-h / p, \varphi_{k}\right\rangle_{\nu, p}^{2} .
\end{aligned}
$$

Since the function $t /(t-1)$ is increasing for $t<1$ and decreasing for $t>1$, the inequalities (2.32) together with the last inequality and Parseval's identity (2.14) yield

where

$$
B(v, v) \leqq(L / \delta)\langle-h / p,-h / p\rangle_{0, p},
$$

$$
L=\max \left[\frac{\alpha_{N} / \gamma_{N}}{1-\alpha_{N} / \gamma_{N}}, \frac{\alpha_{N+1} / \gamma_{N+1}}{\alpha_{N+1} / \gamma_{N+1}-1}\right]
$$

Thus if $M=L / \gamma_{N} \delta$, then $M$ is independent of $p \in \mathscr{P}\left(\gamma_{N}, \gamma_{N+1}\right)$, and since

$$
\langle-h / p,-h / p\rangle_{0, p}=\int_{D} p \frac{h^{2}}{p^{2}} d x \leqq \frac{1}{\gamma_{N}} \int_{D} h^{2} d x
$$

we obtain (2.28). This proves the lemma.

3. A nonlinear problem. In this section $\gamma_{N}$ and $\gamma_{N+1}$ will have the same meaning as in Lemma 2.3. We will assume that $p\left(x, r, s_{1}, \cdots, s_{n}\right)$ and $h\left(x, r, s_{1}, \cdots, s_{n}\right)$ are real valued functions defined and continuous on $\bar{D} \times R^{n+1}$,

$$
\gamma_{N} \leqq p\left(x, r, s_{1}, \cdots, s_{n}\right) \leqq \gamma_{N+1}
$$

for all $\left(x, r, s_{1}, \cdots, s_{n}\right) \in \bar{D} \times R^{n+1}$, and for some constant $L$

$$
\left|h\left(x, r, s_{1}, \cdots, s_{n}\right)\right| \leqq L
$$

on $\bar{D} \times R^{n+1}$.

THEOREM 3.1. Under conditions (3.1) and (3.2) there exists a weak solution of the boundary value problem

$$
\begin{gathered}
L u+p\left(x, u, \frac{\partial u}{\partial x_{1}}, \cdots, \frac{\partial u}{\partial x_{n}}\right) u=h\left(x, u, \frac{\partial u}{\partial x_{1}}, \cdots, \frac{\partial u}{\partial x_{n}}\right), \\
u=0 \text { on } \partial D .
\end{gathered}
$$

(Here $\left(\partial u / \partial x_{k}\right), k=1, \cdots, n$ denote the strong $L^{2}(D)$ derivatives of $u$, i.e., there exists a sequence $\left\{u_{m}\right\}$ in $\dot{C}_{1}$ such that

$$
\left|u-u_{m}\right|_{0} \rightarrow 0,\left|\frac{\partial u}{\partial x_{k}}-\frac{\partial u_{m}}{\partial x_{k}}\right|_{0} \rightarrow 0, k=1, \cdots, n
$$


as $m \rightarrow \infty$.

To prove Theorem 3.1 we use the well-known Schauder method and two auxiliary lemmas.

If $w \in \stackrel{\circ}{H}_{1}$, then by $(3.1)$ and $(3.2)$

$$
p\left(x, w, \frac{\partial w}{\partial x_{1}}, \cdots, \frac{\partial w}{\partial x_{n}}\right) \in \mathscr{P}\left(\gamma_{N}, \gamma_{N+1}\right), h\left(x, w, \frac{\partial w}{\partial x_{1}}, \cdots, \frac{\partial w}{\partial x_{n}}\right) \in H_{0}
$$

and

$$
\left|h\left(x, w, \frac{\partial w}{\partial x_{1}}, \cdots, \frac{\partial w}{\partial x_{n}}\right)\right|_{0}^{2} \leqq R \equiv L^{2} \text { meas } D
$$

Therefore by Lemma 2.3 there exists a unique $w^{*} \in \stackrel{\circ}{H}_{1}$ such that $w^{*}$ is a weak solution of the problem

$$
\begin{gathered}
L u+p\left(x, w, \frac{\partial w}{\partial x_{1}}, \cdots, \frac{\partial w}{\partial x_{n}}\right) u=h\left(x, w, \frac{\partial w}{\partial x_{1}}, \cdots, \frac{\partial w}{\partial x_{n}}\right), \\
u=0 \text { on } \partial D .
\end{gathered}
$$

Furthermore, we have by (2.28)

$$
B\left(w^{*}, w^{*}\right) \leqq M R \text { for all } w \in \stackrel{\circ}{H}_{1}
$$

We define a mapping $G: \stackrel{\circ}{H}_{1} \rightarrow \stackrel{\circ}{H}_{1}$ such that for $w \in \stackrel{\circ}{H}_{1}, G(w)=w^{*}$ is the unique $\dot{H}_{1}$ weak solution of (3.5). If $S=\left\{u \in \stackrel{\circ}{H}_{1} \mid B(u, u) \leqq M R\right\}$ then since $B$ is an inner product on $\stackrel{\circ}{H}_{1}$ which induces the same topology on $\stackrel{\circ}{H}_{1}$ as $\langle,\rangle_{1}, S$ is a closed, bounded, and convex subset of $H_{1}$. Now according to $(3.6), G(S) \subseteq S$, so if it can be shown that $G$ is a compact mapping and that $G$ is continuous then by Schauder's theorem ([3, p. 131]) there exists a $v \in \stackrel{\circ}{H}_{1}$ such that $G(v)=v$. Consequently $v$ is a weak $\stackrel{\circ}{H}_{1}$ solution of (3.5). Accordingly, Theorem (3.1) will follow from the next two lemmas.

\section{LEMMA 3.1. The mapping $G$ is compact.}

\section{LEMMA 3.2. The mapping $G$ is continuous.}

To prove Lemma 3.1 we show that if $\left\{u_{m}\right\}$ is any sequence in $H_{1}$ then there exists a subsequence $\left\{G\left(u_{m_{k}}\right)\right\}$ of $\left\{G\left(u_{m}\right)\right\}$ which converges in the $\stackrel{\circ}{H}_{1}$ norm defined by the $B$ inner product. Suppose then that $\left\{u_{m}\right\}$ is such a sequence. For convenience we set

$$
\begin{aligned}
f_{m}(x) & =h\left(x, u_{m}, \frac{\partial u_{m}}{\partial x_{1}}, \cdots, \frac{\partial u_{m}}{\partial x_{n}}\right) \\
& -p\left(x, u_{m}, \frac{\partial u_{m}}{\partial x_{1}}, \cdots, \frac{\partial u_{m}}{\partial x_{n}}\right) u_{m}^{*}(x)
\end{aligned}
$$


Using (2.3), (3.1), (3.2) and (3.6) and setting $r=L \sqrt{\text { meas } D}+$ $\gamma_{N+1} \sqrt{M R / K_{1}}$ we obtain the inequality

$$
\left|f_{m}\right|_{0} \leqq r
$$

valid for all $m=1,2, \cdots$. By the way $f_{m}$ is defined, $u_{m}^{*}=G\left(u_{m}\right)$ is the weak solution of the problem

$$
L u=-f_{m}, u=0 \text { on } \partial D,
$$

and hence

$$
B\left(\varphi, u_{m}^{*}\right)=\left\langle\varphi, f_{m}\right\rangle_{0} \text { for all } \varphi \in \stackrel{\circ}{H}_{1} .
$$

By (3.6), $B\left(u_{m}^{*}, u_{m}^{*}\right) \leqq M R$ so by (2.4) the sequence $\left\{u_{m}^{*}\right\}$ is bounded in the $H_{1}$ norm. Using Rellich's selection theorem $([1, p .30])$ we infer the existence of a subsequence $\left\{u_{m_{k}}^{*}\right\}$ of $\left\{u_{m}^{*}\right\}$ which converges in the $H_{0}$ norm.

From (3.8) it follows that for arbitrary integers $p$ and $q$ and arbitrary $\varphi \in \stackrel{\circ}{H}_{1}$,

$$
B\left(\varphi, u_{m_{q}}^{*}-u_{m_{p}}^{*}\right)=\left\langle\varphi, f_{m_{q}}-f_{m_{p}}\right\rangle_{0} .
$$

Thus, taking $\varphi=u_{m_{q}}^{*}-u_{m_{p}}^{*}$ in the above and using the Schwartz inequality and (3.7)

$$
B\left(u_{m_{q}}^{*}-u_{m_{p}}^{*}, u_{m_{q}}^{*}-u_{m_{p}}^{*}\right) \leqq 2 r\left|u_{m_{q}}^{*}-u_{m_{p}}^{*}\right|_{0} .
$$

Thus, since the sequence $\left\{u_{m_{k}}^{*}\right\}$ is Cauchy with respect to the $\mid{ }_{0}$ norm, it follows from (2.4) that $\left\{u_{m_{k}}^{*}\right\}$ is Cauchy with respect to the $\stackrel{\circ}{H}_{1}$ norm and hence converges to a member of $\stackrel{\circ}{H}_{1}$. This proves Lemma 3.1.

The proof of the continuity of the mapping $G$ is less straightforward. We will first show that regarded as a map from $H_{1} \rightarrow H_{0}, G$ is continuous and then apply an argument similar to that given above.

Proposition 3.1. The mappings

$$
u \rightarrow p\left(x, u, \frac{\partial u}{\partial x_{1}}, \cdots, \frac{\partial u}{\partial x_{n}}\right),
$$

and

$$
u \rightarrow h\left(x, u, \frac{\partial u}{\partial x_{1}}, \cdots, \frac{\partial u}{\partial x_{n}}\right)
$$

from $\stackrel{\circ}{H}_{1} \rightarrow H_{0}$ are continuous.

Since the proofs of both assertions are similar, we only prove one. Let $\left\{u_{m}\right\}$ be a sequence in $\stackrel{\circ}{H}_{1}$ and $u$ a member of $\stackrel{\circ}{H}_{1}$ such that 


$$
\left|u-u_{m}\right|_{1}^{2}=\int_{D}\left[\left(u-u_{m}\right)^{2}+\sum_{k=1}^{n}\left(\frac{\partial u}{\partial x_{k}}-\frac{\partial u_{m}}{\partial x_{k}}\right)^{2}\right] d x \rightarrow 0
$$

as $m \rightarrow \infty$. Choose $\varepsilon>0$ and define

$$
R(\varepsilon)=\left\{\left(x, t, s_{1}, \cdots, s_{n}\right) \in \bar{D} \times R^{n+1} \mid t^{2}+\sum_{k=1}^{n} s_{k}^{2} \leqq \frac{1}{\varepsilon^{2}}\right\} .
$$

By the compactness of $R(\varepsilon)$ there exists a number $\delta>0$ such that

$$
\left|p\left(x, t, s_{1}, \cdots, s_{n}\right)-p\left(x, t^{\prime}, s_{1}^{\prime}, \cdots, s_{n}^{\prime}\right)\right| \leqq \varepsilon
$$

if $\left(x, t, s_{1}, \cdots s_{n}\right) \in R(\varepsilon)$ and

$$
\left(t-t^{\prime}\right)^{2}+\sum_{k=1}^{n}\left(s_{k}-s_{k}^{\prime}\right)^{2} \leqq \delta^{2} .
$$

Let

$$
A(\varepsilon)=\left\{x \in D \mid u(x)^{2}+\sum_{k=1}^{n} \frac{\partial u}{\partial x_{k}}(x)^{2}>\frac{1}{\varepsilon^{2}}\right\}
$$

and for each integer $m$ define

$$
B_{m}(\delta)=\left\{x \in D \mid\left(u(x)-u_{m}(x)\right)^{2}+\sum_{k=1}^{m}\left(\frac{\partial u(x)}{\partial x_{k}}-\frac{\partial u_{m}(x)}{\partial x_{k}}\right)^{2}>\delta^{2}\right\} .
$$

Since,

$$
\begin{gathered}
\frac{1}{\varepsilon^{2}} \text { meas } A(\varepsilon) \leqq \int_{A(\varepsilon)}\left[u(x)^{2}+\sum_{k=1}^{m} \frac{\partial u(x)}{\partial x_{k}}\right]^{2}, \\
\text { meas } A(\varepsilon) \leqq \varepsilon^{2}|u|_{1}^{2},
\end{gathered}
$$

and in a similar manner we obtain the estimate

$$
\text { meas } B_{m}(\delta) \leqq\left(1 / \delta^{2}\right)\left|u_{m}-u\right|_{1}^{2} \text {. }
$$

For convenience we set

$$
\begin{gathered}
\hat{p}(x)=p\left(x, u(x), \frac{\partial u(x)}{\partial x_{1}}, \cdots, \frac{\partial u(x)}{\partial x_{n}}\right) \\
\hat{p}_{m}(x)=p_{m}\left(x, u_{m}(x), \frac{\partial u_{m}(x)}{\partial x_{1}}, \cdots, \frac{\partial u_{m}(x)}{\partial x_{n}}\right) .
\end{gathered}
$$

If $x \in D-\left(A(\varepsilon) \cup B_{m}(\delta)\right)$ then $\left|\hat{p}(x)-\hat{p}_{m}(x)\right|^{2} \leqq \varepsilon^{2}$, so by the above

$$
\begin{aligned}
\left|\hat{p}-\hat{p}_{m}\right|_{0}^{2} & \leqq \int_{D-\left(A(\varepsilon) \cup B_{m}(\hat{\jmath})\right)}\left|\hat{p}-\hat{p}_{m}\right|^{2} d x+\int_{A(\varepsilon) \cup B_{m}(\delta)}\left|\hat{p}-\hat{p}_{m}\right|^{2} d x \\
& \leqq \varepsilon^{2} \text { meas }\left[D-\left(A(\varepsilon) \cup B_{m}(\delta)\right]+4 \gamma_{N+1}^{2} \text { meas }\left[A(\varepsilon) \cup B_{m}(\delta)\right]\right. \\
& \leqq \varepsilon^{2} \text { meas } D+4 \gamma_{N+1}^{2}\left[\frac{\left|u_{m}-u\right|_{1}^{2}}{\delta^{2}}+\varepsilon^{2}|u|_{1}^{2}\right] .
\end{aligned}
$$


This shows that

$$
\varlimsup_{m \rightarrow \infty}\left|\hat{p}-\hat{p}_{m}\right|_{0}^{2} \leqq \varepsilon^{2}\left[\text { meas } D+4 \gamma_{N+1}^{2}\left|u_{1}\right|^{2}\right]
$$

and since $\varepsilon>0$ is arbitrary, $\lim _{m \rightarrow \infty} \mid \hat{p}-\hat{p}_{m}\left[_{0}^{2}=0\right.$. This proves the continuity of the mapping defined in (3.9) and the proof for (3.10) is similar. In a similar manner one proves that the mapping from $H_{1} \rightarrow H_{0}$ defined by

$$
u \rightarrow \frac{h\left(x, u, \frac{\partial u}{\partial x_{1}}, \cdots, \frac{\partial u}{\partial x_{n}}\right)}{p\left(x, u, \frac{\partial u}{\partial x_{1}}, \cdots, \frac{\partial u}{\partial x_{n}}\right)}
$$

is continuous.

Proposition 3.2. Let $\left\{\hat{p}_{m}\right\}$ be a sequence in $\mathscr{P}\left(\gamma_{N}, \gamma_{N+1}\right)$ and suppose $\left|\hat{p}_{m}-\hat{p}\right|_{0} \rightarrow 0$ as $m \rightarrow \infty$ for some $\hat{p} \in \mathscr{P}\left(\gamma_{N}, \gamma_{N+1}\right)$. If $\left\{T_{\hat{p}_{m}}\right\}$ and $T_{\hat{p}}$ are the operators defined by (2.7) then $T_{\hat{p}_{m}}$ converges strongly to $T_{\hat{p}}$, i.e., $\left|T_{\hat{p}_{m}} w-T_{\hat{p}} w\right|_{0} \rightarrow 0$ as $m \rightarrow \infty$ for each $w \in H_{0}$.

Proof. According to (2.7) if $w \in H_{0}$ and $\varphi \in \stackrel{\circ}{H}_{1}$ then

$$
B\left(\varphi, T_{\hat{p}} w-T_{\hat{p}_{m}} w\right)=\int_{D}\left(\hat{p}_{m}-\hat{p}\right) w \varphi d x .
$$

Now $\lim _{m \rightarrow \infty}\left|\hat{p}_{m}-\hat{p}\right|_{0}^{2}=0$ implies that $\hat{p}_{m}$ converges to $\hat{p}$ in measure. Thus, since $\left|\left(p_{m}(x)-\hat{p}(x)\right) w(x) \varphi(x)\right| \leqq 2 \gamma_{N+1}|w(x) \varphi(x)|$ and $w \varphi \in L^{1}(D)$, by the strong form of Lebesgues' dominated convergence theorem [6, p. 149], $\lim _{m \rightarrow \infty} \int_{D}\left(\hat{p}_{m}-\hat{p}\right) w \varphi d x=0$. This shows that $T_{\hat{p}} w \rightarrow T_{\hat{p}} w$ as $m \rightarrow \infty$ weakly in $H_{1}$ and hence by Rellich's theorem $\lim _{m \rightarrow \infty} T_{\hat{p}_{m}} w=T_{\hat{p}} w$ strongly in $H_{0}$.

Proposition 3.3. The mapping $u \rightarrow G(u)$ is continuous from $H_{0} \rightarrow \stackrel{\circ}{H}_{1}$.

Proof. If $p \in \mathscr{G}\left(\gamma_{N}, \gamma_{N+1}\right)$ let $\left\|T_{p}\right\|_{0}$ and $\left\|T_{p}\right\|_{0, p}$ denote the norms of $T_{p}: H_{0} \rightarrow H_{0}$ relative to the inner products $\langle,\rangle_{0}$ and $\langle,\rangle_{0, p}$ respectively. The identity (2.11) and Lemma 2.2 gives the inequality $\left\|T_{p}\right\|_{0, p} \leqq 1 / \lambda_{1}(p) \leqq 1 / \lambda_{1}\left(\gamma_{N+1}\right)$. Similarly (2.12) and (2.33) give

$$
\left\|\left[I-T_{p}\right]^{-1}\right\|_{0, p} \leqq 1+1 / \delta .
$$

Therefore from the inequality $\langle w, w\rangle_{0}^{2} \leqq 1 / \gamma_{N}\langle w, w\rangle_{0, p}$ valid for any $w \in H_{0}$, we have the estimates

$$
\left\|T_{p}\right\|_{0} \leqq\left(1 / \sqrt{\gamma_{N}}\right)\left(1 / \lambda_{1}\left(\gamma_{N+1}\right)\right) \equiv A_{1}
$$




$$
\left\|\left[I-T_{p}\right]^{-1}\right\|_{0} \leqq\left(1 / \sqrt{\gamma_{N}}\right)(1+1 / \delta) \equiv A_{2} .
$$

Let $u \in \stackrel{\circ}{H}_{1}$ and suppose $\left\{u_{m}\right\}$ is a sequence in $\dot{H}_{1}$ such that $\left|u-u_{m}\right|_{1} \rightarrow 0$ as $m \rightarrow \infty$. Let $\hat{p}$ and $\hat{p}_{m}$ be defined as in (3.11), (3.12) and set

$$
\hat{h}_{m}=h\left(x, u_{m}, \frac{\partial u_{m}}{\partial x_{1}}, \cdots, \frac{\partial u_{m}}{\partial x_{k}}\right), \quad \hat{h}=h\left(x, u, \frac{\partial u}{\partial x_{1}}, \cdots, \frac{\partial u}{\partial x_{n}}\right) .
$$

Let $u^{*}=G(u)$ and $u_{m}^{*}=G\left(u_{m}\right)$. For each $m, u_{m}^{*}$ is the weak solution of the boundary value problem

$$
L v=-\left(\hat{p}_{m} u_{m}^{*}-h_{m}\right), \quad v=0 \text { on } \partial D
$$

so for arbitrary $\varphi \in \stackrel{\circ}{H}_{1}$, and hence

$$
B\left(\varphi, u_{m}^{*}\right)=\left\langle\varphi, \hat{p}_{m} u_{m}^{*}-h_{m}\right\rangle_{0}=\left\langle\varphi, u_{m}^{*}-h_{m} / \hat{p}_{m}\right\rangle_{0, \hat{p}_{m}}
$$

$$
\begin{gathered}
u_{m}^{*}=T_{\hat{p}_{m}}\left[u_{m}^{*}-\hat{h}_{m} / \hat{p}_{m}\right], \\
u^{*}=T_{\hat{p}}\left[u^{*}-\hat{h} / \hat{p}\right] .
\end{gathered}
$$

From the equation

$$
\begin{aligned}
u^{*}-u_{m}^{*}= & T_{\hat{p}_{m}}\left[u^{*}-u_{m}^{*}\right]+\left[T_{\hat{p}}-T_{\hat{p}_{m}}\right] u^{*} \\
& +T_{\hat{p}_{m}}\left[\hat{h}_{m} / \hat{p}_{m}-\hat{h} / \hat{p}\right]+\left[T_{\hat{p}_{m}}-T_{\hat{p}}\right] \hat{h} / \hat{p},
\end{aligned}
$$

we obtain

$$
\begin{aligned}
u^{*}- & u_{m}^{*} \\
& =\left[I-T_{\hat{p}_{m}}\right]^{-1}\left(T_{\hat{p}}-T_{\hat{p}_{m}}\right)\left[u^{*}-\hat{h} / \hat{p}\right]+\left[I-T_{\hat{p}_{m}}\right]^{-1} T_{\hat{p}_{m}}\left[\hat{h}_{m} / \hat{p}_{m}-\hat{h} / \hat{p}\right] .
\end{aligned}
$$

Therefore by the estimates (3.14), (3.15) we have

$$
\begin{aligned}
\left|u^{*}-u_{m}^{*}\right|_{0} \leqq A_{2}\left|\left(T_{\hat{p}}-T_{\hat{p}_{m}}\right)\left(u^{*}-\hat{h} / \hat{p}\right)\right|_{0} \\
+A_{2} A_{1}\left|\hat{h}_{m} / \hat{p}_{m}-\hat{h} / \hat{p}\right|_{0} .
\end{aligned}
$$

By Propositions 3.1 and 3.2,

$$
\left|\left(T_{\hat{p}}-T_{\hat{p}_{m}}\right)\left(u^{*}-\hat{h} / \hat{p}\right)\right|_{0} \rightarrow 0 \text { as } m \rightarrow \infty,
$$

and by the remark following the proof of Proposition 3.1,

$$
\left|\hat{h}_{m} / \hat{p}_{m}-\hat{h} / \hat{p}\right|_{0} \rightarrow 0 \text { as } m \rightarrow \infty \text {. }
$$

This concludes the proof of Proposition 3.

Lemma 3.2 now follows easily. Let $u$ and the sequence $u_{m}$ be as above. Define $f_{m}=\hat{h}_{m}-\hat{p}_{m} u_{m}^{*}, f=\hat{h}-\hat{p} u^{*}$. From (3.7) $\left|f_{m}\right|_{0} \leqq r$, $|f|_{0} \leqq r$. Referring to the proof of Lemma 3.1 we see that for any $\varphi \in \stackrel{\circ}{H}_{1}$

$$
B\left(\varphi, u^{*}-u_{m}^{*}\right)=\left\langle\varphi, f-f_{m}\right\rangle_{0},
$$


so by taking $\varphi=u^{*}-u_{m}^{*}$ we have

$$
\begin{aligned}
B\left(u^{*}-u_{m}^{*}, u^{*}-u_{m}^{*}\right) & =\left\langle u^{*}-u_{m}^{*}, f-f_{m}\right\rangle_{0} \\
& \leqq 2\left|u^{*}-u_{m}\right|_{0} r .
\end{aligned}
$$

By Proposition 3.3, $B\left(u^{*}-u_{m}^{*}, u^{*}-u_{m}^{*}\right) \rightarrow 0$ as $m \rightarrow \infty$. This proves the continuity of $G: \stackrel{\circ}{H}_{1} \rightarrow \stackrel{\circ}{H}_{1}$ and concludes the proof of Theorem 3.1.

4. Smooth solutions of an inhomogeneous problem. In this section we will assume that $D \subset R^{n}$ is a Dirichlet domain and $L=\Delta$ where $\Delta$ is the $n$-dimensional Laplacian.

If $f$ is continuous on $\bar{D}$ and has continuous partial derivatives on $D$, then the weak solution of the problem

$$
\Delta u=-f(x), \quad u=0 \text { on } \partial D
$$

is actually a solution in the classical sense and can be represented in the form

$$
u(y)=\int_{D} G(x, y) f(x) d x, \quad y \in \bar{D},
$$

where $G$ is the Green's function for the problem (4.1).

THEOREM 4.1. If $p$ and $h$ satisfy the conditions (3.1) and (3.2) of Theorem 3.1 and $g$ is continuous on $\partial D$, then there exists a weak solution $v$ of

$$
\Delta u+p\left(x, u, \frac{\partial u}{\partial x_{1}}, \cdots, \frac{\partial u}{\partial x_{n}}\right) u=h\left(x, u, \frac{\partial u}{\partial x_{1}}, \cdots, \frac{\partial u}{\partial x_{n}}\right)
$$

such that $v$ has continuous derivatives on $\bar{D}$ and

$$
v(x)=g(x), \quad x \in \partial D .
$$

Proof. Since $D$ is a Dirichlet domain there exists a function $w$ such that $w$ is continuous on $\bar{D}, \Delta w=0$ on $D$, and $w(x)=g(x)$ on $\partial D$. If

$$
\begin{aligned}
& P\left(x, t, s_{1}, \cdots, s_{n}\right) \\
& =p\left(x, t+w(x), s_{1}+\frac{\partial w(x)}{\partial x_{1}}, \cdots, s_{n}+\frac{\partial w(x)}{\partial x_{n}}\right) \\
& H\left(x, t, s_{1}, \cdots, s_{n}\right) \\
& =h\left(x, t+w(x), s_{1}+\frac{\partial w(x)}{\partial x_{1}}, \cdots, s_{n}+\frac{\partial w(x)}{\partial x_{n}}\right) \\
& \quad-P\left(x, t, s_{1}, \cdots, s_{n}\right) w(x),
\end{aligned}
$$


then $P$ and $H$ will satisfy conditions of the form (3.1) and (3.2).

Consequently, by Theorem 3.1 there exists a weak $\stackrel{\circ}{H}_{1}$ solution $V$ of

$$
\Delta u+P\left(x, u, \frac{\partial u}{\partial x_{1}}, \cdots, \frac{\partial u}{\partial x_{n}}\right) u=H\left(x, u, \frac{\partial u}{\partial x_{1}}, \cdots, \frac{\partial u}{\partial x_{n}}\right) .
$$

We assert that if $y \in \bar{D}$,

$$
V(y)=\int_{D} G(x, y) F(x) d x
$$

where as in (4.2), $G$ is the Green's function and

$$
F=P\left(x, V, \frac{\partial V}{\partial x_{1}}, \cdots, \frac{\partial V}{\partial x_{n}}\right) V-H\left(x, V, \frac{\partial V}{\partial x_{1}}, \cdots, \frac{\partial V}{\partial x_{n}}\right) .
$$

Indeed, $V$ is the weak $\stackrel{\circ}{H}_{1}$ solution of $\Delta u=-F$ so $V=T_{1} F$ where $T_{1}$ is defined by (2.7). If $f$ is continuously differentiable on $\bar{D}$ then by (4.2) for $y \in \bar{D}$

$$
\left(T_{1} f\right)(y)=\int_{D} G(x, y) f(x) d x .
$$

Now if $f$ is merely in $L^{2}(D)$, the operator $S: \stackrel{\circ}{H}_{1} \rightarrow \stackrel{\circ}{H}_{1}$ defined by

$$
(S f)(y)=\int_{D} G(x, y) f(x) d x
$$

is continuous. Therefore, since $S$ and $T_{1}$ agree on a dense subspace of $L^{2}(D), T_{1}=S$, whence (4.7) holds.

From the representation (4.7) and the fact that $F$ is in $L^{\infty}(D)$, it follows by standard arguments of potential theory that $V$ has continuous derivatives and vanishes on the boundary of D. Setting $v=V+w$ we see that $v$ satisfies the assertion of Theorem 4.1.

\section{REFERENCES}

1. S. Agmon, Lectures on elliptic boundary value problems, D. Van Nostrand Company, Inc., New York, 1965.

2. R. Courant and D. Hilbert, Methods of mathematical physics, Val. I, Interscience Publishers, Inc., New York, 1953.

3. J. Cronin, Fixed points and topological degree in nonlinear analysis, Mathematical Surveys, No. 11, American Mathematical Society, Providence, 1964.

4. C. L. Dolph, Nonlinear integral equations of the Hammerstein type, Trans. Amer. Math. Soc. 66 (1949), 289-307.

5. A. C. Lazer and D. E. Leach, On a nonlinear two-point boundary value problem, J. Math. Anal. and Appl. 26 (1969), 20-27.

6. I. P. Natanson, Theory of functions of a real variable, Vol. I, (transl. from the Russian), Ungar, New York, 1961.

7. F. Riesz and B. Sz.-Nagy, Functional analysis, (translated from the 2'nd. French edition), Ungar, New York, 1955. 
Received June 27, 1969. The preparation of this paper was sponsored in part by the Office of Naval Research under Contract NONR 233(76) and the U. S. Army Research Office under Grant DA-31-124-ARO(D)-355. Reproduction in whole or in part is permitted for any purpose of the United States Government.

University of California, Los ANgeles, AND

University of California, Santa Cruz

UNiversity of California, Los ANgeles, and

Case Western Reserve University 


\section{PACIFIC JOURNAL OF MATHEMATICS}

\section{EDITORS}

H. SAMelson

Stanford University

Stanford, California 94305

\section{Richard PIERCE}

University of Washington

Seattle, Washington 98105
J. DugundjI

Department of Mathematics

University of Southern California

Los Angeles, California 90007

BASIL GoRDON*

University of California

Los Angeles, California 90024

\section{ASSOCIATE EDITORS}
E. F. BECKENBACH
B. H. NeumanN
F. WOLE
K. YosHIDA

\section{SUPPORTING INSTITUTIONS}

UNIVERSITY OF BRITISH COLUMBIA

CALIFORNIA INSTITUTE OF TECHNOLOGY

UNIVERSITY OF CALIFORNIA

MONTANA STATE UNIVERSITY

UNIVERSITY OF NEVADA

NEW MEXICO STATE UNIVERSITY

OREGON STATE UNIVERSITY

UNIVERSITY OF OREGON

OSAKA UNIVERSITY

UNIVERSITY OF SOUTHERN CALIFORNIA

\author{
STANFORD UNIVERSITY \\ UNIVERSITY OF TOKYO \\ UNIVERSITY OF UTAH \\ WASHINGTON STATE UNIVERSITY \\ UNIVERSITY OF WASHINGTON \\ * * * * \\ AMERICAN MATHEMATICAL SOCIETY \\ CHEVRON RESEARCH CORPORATION \\ TRW SYSTEMS \\ NAVAL WEAPONS CENTER
}

The Supporting Institutions listed above contribute to the cost of publication of this Journal, but they are not owners or publishers and have no responsibility for its content or policies.

Mathematical papers intended for publication in the Pacific Journal of Mathematics should be in typed form or offset-reproduced, (not dittoed), double spaced with large margins. Underline Greek letters in red, German in green, and script in blue. The first paragraph or two must be capable of being used separately as a synopsis of the entire paper. The editorial "we" must not be used in the synopsis, and items of the bibliography should not be cited there unless absolutely necessary, in which case they must be identified by author and Journal, rather than by item number. Manuscripts, in duplicate if possible, may be sent to any one of the four editors. Please classify according to the scheme of Math. Rev. 36, 1539-1546. All other communications to the editors should be addressed to the managing editor, Richard Arens, University of California, Los Angeles, California, 90024.

50 reprints are provided free for each article; additional copies may be obtained at cost in multiples of 50 .

The Pacific Journal of Mathematics is published monthly. Effective with Volume 16 the price per volume (3 numbers) is $\$ 8.00$; single issues, $\$ 3.00$. Special price for current issues to individual faculty members of supporting institutions and to individual members of the American Mathematical Society: $\$ 4.00$ per volume; single issues $\$ 1.50$. Back numbers are available.

Subscriptions, orders for back numbers, and changes of address should be sent to Pacific Journal of Mathematics, 103 Highland Boulevard, Berkeley, California, 94708.

PUBLISHED BY PACIFIC JOURNAL OF MATHEMATICS, A NON-PROFIT CORPORATION

Printed at Kokusai Bunken Insatsusha (International Academic Printing Co., Ltd.), 7-17, Fujimi 2-chome, Chiyoda-ku, Tokyo, Japan.

* Acting Managing Editor. 


\section{Pacific Journal of Mathematics}

\section{Vol. 33, No. $2 \quad$ April, 1970}

Raymond Balbes and Alfred Horn, Projective distributive lattices ....... 273

John Findley Berglund, On extending almost periodic functions ......... 281

Günter Krause, Admissible modules and a characterization of reduced left

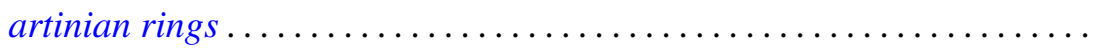

Edward Milton Landesman and Alan Cecil Lazer, Linear eigenvalues and a nonlinear boundary value problem ....................... 311

Anthony To-Ming Lau, Extremely amenable algebras ............... 329

Aldo Joram Lazar, Sections and subsets of simplexes .............. 337

Vincent Mancuso, Mesocompactness and related properties ............. 345

Edwin Leroy Marsden, Jr., The commutator and solvability in a generalized orthomodular lattice .................................. 357

Shozo Matsuura, Bergman kernel functions and the three types of canonical domains.......................................... 363

S. Mukhoti, Theorems on Cesàro summability of series .............. 385

Ngô Van Quê, Classes de Chern et théorème de Gauss-Bonnet ........... 393

Ralph Tyrrell Rockafellar, Generalized Hamiltonian equations for convex problems of Lagrange ................................ 411

Ken iti Sato, On dispersive operators in Banach lattices ............. 429

Charles Andrew Swanson, Comparison theorems for elliptic differential

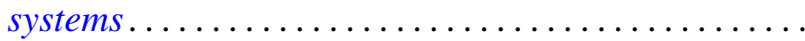

John Griggs Thompson, Nonsolvable finite groups all of whose local subgroups are solvable. II

David J. Winter, Cartan subalgebras of a Lie algebra and its ideals ... 